\title{
Comparison of Helicobacter pylori Eradication Rates of 2-Week Levofloxacin-Containing Triple Therapy, Levofloxacin-Containing Bismuth Quadruple Therapy, and Standard Bismuth Quadruple Therapy as a First-Line Regimen
}

\author{
Evrim Kahramanoğlu Aksoy ${ }^{a}$ Ferdane Pirinçci Sapmaz ${ }^{a}$ Zeynep Göktaş ${ }^{b}$ \\ Metin Uzmana ${ }^{a}$ Yaşar Nazlıgüla \\ ${ }^{a}$ Department of Gastroenterology, Keçiören Training and Research Hospital, and ${ }^{b}$ Department of Nutrition and \\ Dietetics, Faculty of Health Sciences, Hacettepe University, Ankara, Turkey
}

\section{Significance of the Study}

- This study compared the efficacy of 3 Helicobacter pylori eradication regimens, 2 of which contained levofloxacin, as the first line of treatment. Both quadruple regimens (standard bismuth quadruple therapy or levofloxacin-containing bismuth quadruple therapy) were highly effective in eradicating the infection and significantly superior to levofloxacin-containing triple therapy. Quadruple therapies should be used as first-line therapy for $H$. pylori infection.

\section{Keywords}

Helicobacter pylori infection · Bismuth-containing quadruple therapy - Levofloxacin-containing triple therapy · Eradication rate

\section{Abstract \\ Objective: The aim of this study was to compare the efficacy and safety of 2-week levofloxacin-containing triple therapy, levofloxacin-containing bismuth quadruple therapy, and standard bismuth-containing quadruple therapy as a first- line regimen for the eradication of Helicobacter pylori. Meth- ods: A total of 329 patients with H. pylori infection were ran- domly divided into 3 groups to receive one of the following regimens: (a) levofloxacin-containing bismuth quadruple}

\section{KARGER}

E-Mail karger@karger.com www.karger.com/mpp

\section{The Author(s) \\ Published by S. Karger AG, Basel \\ Karger \\ Open access}

This is an Open Access article licensed under the Creative Commons Attribution-NonCommercial-4.0 International License (CC BY-NC) (http://www.karger.com/Services/OpenAccessLicense), applicable to the online version of the article only. Usage and distribution for commercial purposes requires written permission. therapy, RBAL (rabeprazole 20 mg, b.i.d., bismuth subsalicylate 562 mg, b.i.d., amoxicillin 1 g, b.i.d, levofloxacin 500 mg, once daily), (b) standard bismuth quadruple therapy, RBMT (rabeprazole 20 mg, b.i.d, subsalicylate 562 mg, b.i.d., metronidazole 500 mg, t.i.d, tetracycline 500 mg, q.i.d), or (c) levofloxacin-containing triple therapy, RAL (rabeprazole $20 \mathrm{mg}$, b.i.d., amoxicillin $1 \mathrm{~g}$, b.i.d, levofloxacin $500 \mathrm{mg}$, once daily). The primary outcome was the eradication rate in the intention-to-treat (ITT) and per protocol (PP) analysis. Results: The eradication rates of the above 3 groups using ITT analysis were RBAL $83.8 \%$, RBMT $88.3 \%$, and RAL $74.8 \%$ compared with 91.2, 92.5, and 79.2\%, respectively, using PP analysis. The eradication rate using RBMT was significantly higher than that of $\operatorname{RAL}(p=0.029$ in ITT analysis and $p=0.017$ in PP analysis). Several side effects occurred in 156 patients (54.1\%) 
in the RBAL group, $215(52.3 \%)$ in the RBMT group, and 56 (26.2\%) in the RAL group ( $p>0.05$, RBAL vs. RBMT; $p<0.001$, RBMT vs. RAL; $p<0.001$, RBAL vs. RAL). Conclusion: All bismuth-containing quadruple therapies had acceptable eradication rates, but levofloxacin-containing triple therapy was not as good as quadruple therapies. Hence, quadruple therapies should be considered the preferred first-line therapy for $H$. pylori infections.

(C) 2017 The Author(s)

Published by S. Karger AG, Basel

\section{Introduction}

Despite the fact that the presence of Helicobacter $p y$ lori and the diseases it causes, such as gastroduodenal ulcers, gastritis, gastric adenocarcinoma, mucosa-associated lymphoid tissue lymphoma, and nonulcer dyspepsia, have been known for over 30 years, treatment option(s) remain a challenge $[1,2]$. Eighty percent of the population in developing countries and $20-50 \%$ of the population in the developed countries are estimated to carry this pathogen [3]. Eradication of H. pylori infection has been recommended as an effective approach for curing or preventing these $H$. pylori-associated diseases [4]. Standard triple therapy no longer provides the targeted treatment success. In countries with $25-30 \%$ clarithromycin resistance, the eradication rates declined significantly from 90 to $60-70 \%$ in $2006[5,6]$. Although there might be many reasons for the decrease of efficacy of standard triple therapy, such as compliance, bacterial load, host CYP2C19 polymorphisms, and gastric acidity, the most important reason is the increase in $H$. pylori resistance to clarithromycin $[7,8]$.

In high $(>15 \%)$ clarithromycin resistance areas, bismuth quadruple or nonbismuth quadruple concomitant therapies are recommended [9]. In regions of high (>15\%) dual clarithromycin and metronidazole resistance ( $>40 \%$ ), bismuth-containing quadruple therapies are the treatment of choice [9]. Although metronidazole resistance in Europe has stabilized at $34.9 \%$ of $H$. pylori isolates, and the eradication rate can be improved by prolonging the duration of treatment from 10 to 14 days, some studies have shown inefficacy of these treatments $[8,10]$. Based on the recent systematic review of the prevalence (from 1999 to 2015) of primary antibiotic resistance of $H$. pylori strains in different geographical regions of Turkey, the overall primary antibiotic resistance rates of $H$. pylori strains were as follows: amoxicillin $0.971 \%$, clarithromycin $24.864 \%$, metronidazole $33.747 \%$, tetracycline $3.511 \%$, and levofloxacin $23.769 \%$. There was an increase in primary resistance rates to clarithromycin and metronidazole in different years [11].

Levofloxacin is a fluoroquinolone with a broad spectrum of activity both against gram-positive and gramnegative bacteria [12]. The efficacy of levofloxacin-containing triple therapy as second- and third-line therapy has been shown in various studies [12,13]. Furthermore, there are studies showing levofloxacin-containing triple therapy as an effective first-line treatment $[14,15]$. There are controversial data in comparing the efficacy and duration of bismuth-containing quadruple therapies and levofloxacin-containing triple therapies [16, 17]. Some studies showed the efficacy of 1-week bismuth- and levofloxacin-containing quadruple therapies for first-line therapy. However, in other studies the ineffectiveness of these treatment regimens as second-line therapy were pointed out $[16,17]$.

Therefore, in this study, the aim was to determine the efficacy, side effects, and tolerability of a 2-week levofloxacin-containing triple therapy, levofloxacin-containing bismuth quadruple therapy, and standard bismuthcontaining quadruple therapy as a first-line regimen in Turkey.

\section{Subjects and Methods}

\section{Study Population}

A total of 329 patients infected with $H$. pylori were enrolled in this prospective, open-label, randomized study conducted at the Gastroenterology Outpatient Clinic, Keçiören Training and Research Hospital, Ankara, Turkey, from October 2016 to April 2017. The $H$. pylori infection was diagnosed by histological analysis (2 samples from the antrum and 1 sample from the corpus) using Giemsa and hematoxylin and eosin stainings. Patients (aged between 18 and 70 years) who underwent endoscopy due to recurrent dyspeptic symptoms (epigastric pain, epigastric fullness, epigastric dullness, nausea, vomiting, quick saturation) or alarm symptoms and tested positive for $H$. pylori, and who were not previously treated for $H$. pylori infection, were included in the study. Exclusion criteria were $\mathrm{H}_{2}$ receptor antagonists, bismuth preparations, proton pump inhibitors, and antibiotic use up to 4 weeks before upper endoscopy, malignant or severe disease, gastric surgery, pregnant or lactating women, and known allergy to antibiotics.

The study protocol was approved by the Institutional Ethics Committee, and the study was performed in accordance with good clinical practice and the Declaration of Helsinki. Written informed consent was obtained from each participant.

\section{Treatment Protocols}

Random allocation of patients to 3 treatment groups (using a random-numbers table) was done by a gastroenterologist (E.K.A.) to receive one of the following regimens: (a) levofloxacin-containing bismuth quadruple therapy, RBAL (rabeprazole $20 \mathrm{mg}$, b.i.d., bismuth subsalicylate $562 \mathrm{mg}$, b.i.d., amoxicillin $1 \mathrm{~g}$, b.i.d, levoflox- 
Fig. 1. Flow diagram of the study. RBAL, rabeprazole $20 \mathrm{mg}$, b.i.d., bismuth subsalicylate $562 \mathrm{mg}$, b.i.d., amoxicillin $1 \mathrm{~g}$, b.i.d, levofloxacin $500 \mathrm{mg}$, once daily; RBMT, rabeprazole $20 \mathrm{mg}$, b.i.d, subsalicylate $562 \mathrm{mg}$, b.i.d., metronidazole $500 \mathrm{mg}$, t.i.d, tetracycline $500 \mathrm{mg}$, q.i.d.; RAL, rabeprazole $20 \mathrm{mg}$, b.i.d., amoxicillin $1 \mathrm{~g}$, b.i.d, levofloxacin $500 \mathrm{mg}$, once daily; ITT, intention-to-treat; PP, per protocol.

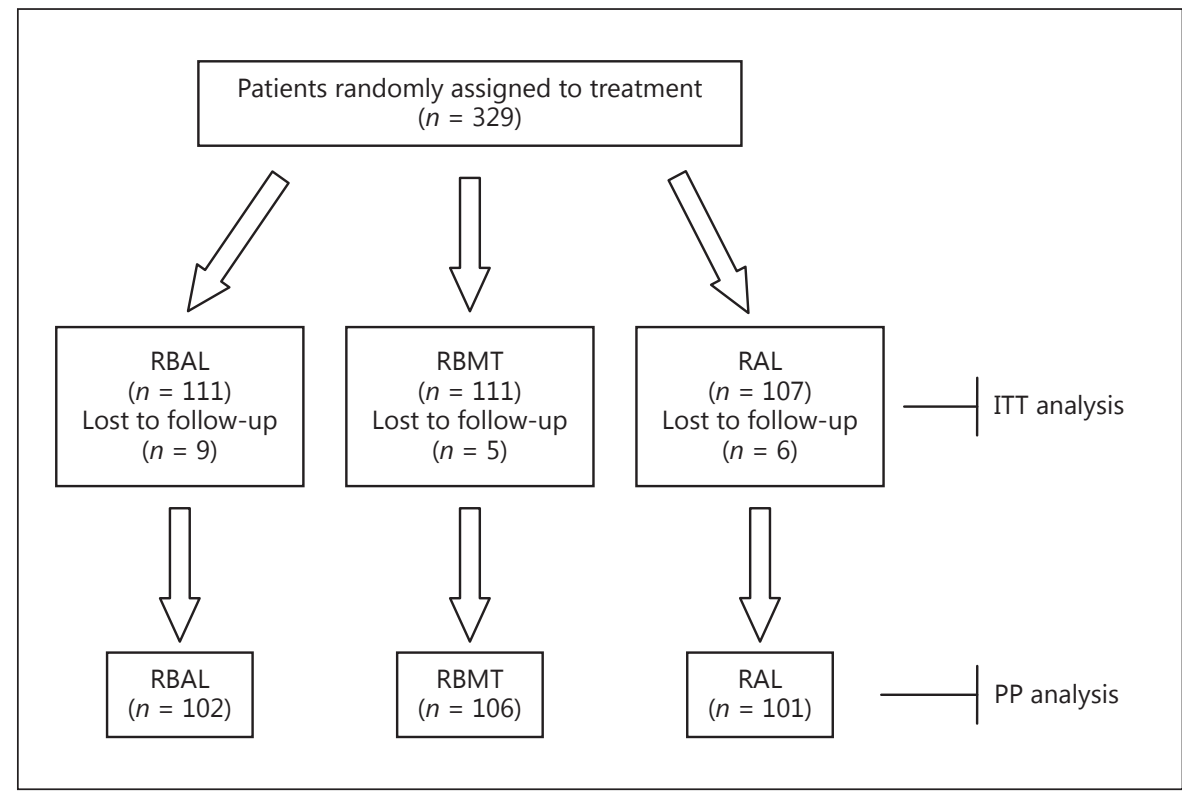

Table 1. Baseline demographic and endoscopic data

\begin{tabular}{|c|c|c|c|c|}
\hline & RBAL & RBMT & RAL & $p$ value \\
\hline Patients (ITT) & 111 & 111 & 107 & $>0.05$ \\
\hline Gender (male/female) & $37 / 64$ & $45 / 56$ & $42 / 59$ & $>0.05$ \\
\hline Age, years & $49.1 \pm 14.54$ & $45.3 \pm 12.71$ & $47.9 \pm 13.99$ & $>0.05$ \\
\hline Smoking habit (yes/no) & $27 / 74$ & $24 / 77$ & $25 / 76$ & $>0.05$ \\
\hline \multicolumn{5}{|l|}{ Endoscopic diagnosis } \\
\hline Gastritis & $109(98.2)$ & $107(96.4)$ & $107(100)$ & $>0.05$ \\
\hline Gastric ulcer & $2(1.8)$ & $6(5.4)$ & $1(0.9)$ & $>0.05$ \\
\hline Duodenal ulcer & $5(4.5)$ & $10(9.0)$ & $2(1.9)$ & $>0.05$ \\
\hline Esophagitis & $10(9.0)$ & $9(8.1)$ & $4(3.7)$ & $>0.05$ \\
\hline Duodenitis & $27(24.3)^{\mathrm{a}}$ & $31(27.9)^{\mathrm{a}}$ & $11(10.3)^{b}$ & $0.006,0.001^{*}$ \\
\hline
\end{tabular}

Data are presented as $n(\%)$ or mean \pm SD, as appropriate. RBAL, rabeprazole $20 \mathrm{mg}$, b.i.d., bismuth subsalicylate 562 mg, b.i.d., amoxicillin $1 \mathrm{~g}$, b.i.d, levofloxacin $500 \mathrm{mg}$, once daily; RBMT, rabeprazole $20 \mathrm{mg}$, b.i.d, subsalicylate $562 \mathrm{mg}$, b.i.d., metronidazole 500 $\mathrm{mg}$, t.i.d, tetracycline $500 \mathrm{mg}$, q.i.d.; RAL, rabeprazole $20 \mathrm{mg}$, b.i.d., amoxicillin $1 \mathrm{~g}$, b.i.d, levofloxacin $500 \mathrm{mg}$, once daily; ITT, intentionto-treat. ${ }^{*} p=0.006$, RBAL vs. RAL; $p=0.001$, RBMT vs. RAL; $p>0.05$, RBAL vs. RBMT. ${ }^{\text {a }}$ b Protocols within a row without a common superscript differ.

acin $500 \mathrm{mg}$, once daily, for 2 weeks) ( $n=111$ patients), (b) standard bismuth-containing quadruple therapy, RBMT (rabeprazole 20 mg, b.i.d, subsalicylate $562 \mathrm{mg}$, b.i.d., metronidazole $500 \mathrm{mg}$, t.i.d, tetracycline $500 \mathrm{mg}$, q.i.d, for 2 weeks) ( $n=111$ patients), or (c) levofloxacin-containing triple therapy, RAL (rabeprazole $20 \mathrm{mg}$, b.i.d., amoxicillin $1 \mathrm{~g}$, b.i.d, levofloxacin $500 \mathrm{mg}$, once daily, for 2 weeks) ( $n=107$ patients). H. pylori eradication was determined using the stool antigen test with an enzyme immunoassay utilizing a monoclonal antibody. The test was performed at least 4 weeks after the end of therapy. Patients were asked to avoid antacid treatment and antibiotics for 1 month before the stool antigen test.

Helicobacter pylori Treatment
A questionnaire was used to record side effects for all patients. In the RBAL group, 106 of 111 patients completed the course and 5 patients failed to take at least $75 \%$ of the drugs. In the RBMT group, 102 of 111 patients completed the course and 9 patients failed to take at least $75 \%$ of the drugs. In the RAL group, 101 of 107 patients completed the course and 6 patients failed to take at least $75 \%$ of the drugs. Twenty patients (5 in RBAL, 9 in RBMT, and 6 in RAL therapy groups) withdrew from the treatment due to nausea, dysphagia, drowsiness, insomnia, headache/dizziness, chest pain, and skin allergy. A flow diagram of the study is presented in Figure 1. 
Table 2. Helicobacter pylori eradication rates with different treatment regimens

\begin{tabular}{lllll}
\hline & RBAL & RBMT & RAL & $p$ value \\
\hline ITT analysis & $93 / 111(83.8)^{\mathrm{a}, \mathrm{b}}$ & $98 / 111(88.3)^{\mathrm{a}}$ & $80 / 107(74.8)^{\mathrm{b}}$ & $0.029^{*}$ \\
$\quad$ 95\% CI & $76.9-90.7$ & $82.3-94.2$ & $72.7-76.9$ & \\
\hline Per protocol analysis & $93 / 102(91.2)^{\mathrm{a}, \mathrm{b}}$ & $98 / 106(92.5)^{\mathrm{a}}$ & $80 / 101(79.2)^{\mathrm{b}}$ & $0.017^{* *}$ \\
$\quad$ 95\% CI & $85.7-96.7$ & $87.5-97.5$ & $71.3-87.1$ & \\
\hline Compliance & $106 / 111(95.5)$ & $102 / 111(91.9)$ & $101 / 107(94.4)$ & $>0.05$ \\
95\% CI & $91.6-99.4$ & $86.8-96.9$ & $90.1-98.8$ & \\
\hline
\end{tabular}

Data are presented as $n(\%)$ with 95\% CI. See Table 1 footnote for abbreviations. $* p=0.029$, RBMT vs. RAL; $p>0.05$, RBAL vs. RBMT; $p>0.05$, RBAL vs. RAL. ${ }^{* *} p=0.017$, RBMT vs. RAL; $p>0.05$, RBAL vs. RBMT; $p>$ 0.05 , RBAL vs. RAL. ${ }^{\text {a, b }}$ Protocols within a row without a common superscript differ.

\section{Statistical Analysis}

Statistical analyses were performed using the computer program Statistical Package for the Social Sciences (SPSS), version 22.0 (IBM, Armonk, NY, USA). The Levene test was used to test for equality of variances between arms. One-way ANOVA was used for comparison of more than 2 group means. The $\chi^{2}$ test was used to compare eradication rates, side effects, and symptoms between different treatment groups. In the assessment of treatment, intention-to-treat (ITT) and per protocol (PP) analyses were used. All of the participants were included in the ITT analysis. Participants who did not follow the study protocol or dropped out of the study were excluded from the PP analysis. Data are presented as the mean \pm standard deviation or number and percentage. Differences were considered significant at $p<0.05$.

\section{Results}

\section{Demographic Data}

Relevant demographic and endoscopic data are given in Table 1. There was no difference between groups in terms of age, sex, and smoking habits $(p>0.05)$.

\section{Eradication Rates}

The efficacy of the eradication regimens is shown in Table 2. In the ITT analysis, eradication rates were $93 / 111$ (83.8\%; 95\% CI, 76.9-90.7) with RBAL, 98/111 (88.3\%; 95\% CI, 82.3-94.2) with RBMT, and 80/107 (74.8\%; 95\% CI, 72.7-76.9\%) with RAL. In the PP analysis, eradication rates were 93/86 (91.2\%; 95\% CI, 85.7-96.7\%) with RBAL, 98/106 (92.5\%; 95\% CI, 87.5-97.5\%) with RBMT, and 80/101 (79.2\%; 95\% CI, 71.3-87.1\%) with RAL.

In the comparison of all treatment groups separately with each other based on $H$. pylori eradication rates, there were statistically significant differences between the RBMT and RAL groups for both ITT and PP analyses $(p=0.029$ and $p=0.017$, respectively) (Table 2). There were no statistically significant differences between RBAL and RBMT or between RBAL and RAL groups in terms of eradication rates $(p>0.05)$ (Table 2).

\section{Side Effects}

The most common side effects were darkened stool, nausea, abdominal pain, black tongue, and diarrhea. Several side effects at various degrees occurred in 156 patients $(54.1 \%)$ in the RBAL group, $215(52.3 \%)$ in the RBMT group, and $56(26.2 \%)$ in the RAL group $(p>0.05$, RBAL vs. RBMT; $p<0.001$, RBMT vs. RAL; $p<0.001$, RBAL vs. RAL) (Table 3).

\section{Discussion}

This study showed that both quadruple regimens (standard bismuth quadruple therapy and levofloxacincontaining bismuth quadruple therapy) were highly effective in eradicating the infection (>90\%), and better than levofloxacin-containing triple therapy $(79.2 \%)$, in Turkey, which has one of the highest levels of primary clarithromycin resistance (48.2\%) [18].

The high eradication rates of RBAL (91.2\%) and RBMT $(92.5 \%)$ are consistent with the recommendation of the current guidelines that bismuth-containing quadruple therapies be used as first-line treatment in areas with high resistance to clarithromycin $(>15 \%)[9,19]$. Bismuth salts act topically, and less than $1 \%$ of the oral dose is absorbed. In acidic $\mathrm{pH}$ it forms nonsoluble polymers. In this cytoprotective form, it covers the ulcer crater and prevents it from gastric acidity. Furthermore, it possess antibacterial effects by inhibiting various enzymes produced by $H$. $p y$ - 
Table 3. Side effects of different treatment regiments

\begin{tabular}{|c|c|c|c|c|c|c|}
\hline \multirow[b]{2}{*}{$\begin{array}{l}\text { Side effects of treatment } \\
\quad \text { (yes), } \%\end{array}$} & \multirow{2}{*}{$\begin{array}{l}\text { RBAL } \\
54.1^{\mathrm{a}}\end{array}$} & \multirow{2}{*}{$\begin{array}{l}\text { RBMT } \\
52.3^{\mathrm{a}}\end{array}$} & \multirow{2}{*}{$\begin{array}{l}\text { RAL } \\
26.2^{\mathrm{b}}\end{array}$} & \multicolumn{3}{|l|}{$p$ value* } \\
\hline & & & & $>0.05$ & $<0.001$ & $<0.001$ \\
\hline $\begin{array}{l}\text { Patients withdrawn due to } \\
\text { side effects }\end{array}$ & $5(4.5)$ & $9(8.1)$ & $6(5.6)$ & $>0.05$ & $>0.05$ & $>0.05$ \\
\hline \multicolumn{7}{|l|}{ Side effects } \\
\hline Nausea & $37(33.3)^{\mathrm{a}}$ & $45(40.5)^{\mathrm{a}}$ & $13(12.1)^{\mathrm{b}}$ & $>0.05$ & $<0.001$ & $<0.001$ \\
\hline Vomiting & $6(5.4)^{\mathrm{a}}$ & $11(9.9)^{\mathrm{a}}$ & $0(0.0)^{\mathrm{b}}$ & $>0.05$ & $<0.001$ & 0.015 \\
\hline Metallic taste & $0(0.0)^{\mathrm{a}}$ & $23(20.7)^{\mathrm{b}}$ & $0(0.0)^{\mathrm{a}}$ & $<0.001$ & $<0.001$ & $>0.05$ \\
\hline Itchiness & $8(7.2)$ & $6(5.4)$ & $5(4.7)$ & $>0.05$ & $>0.05$ & $>0.05$ \\
\hline Skin rash & $5(4.5)$ & $4(3.6)$ & $7(6.5)$ & $>0.05$ & $>0.05$ & $>0.05$ \\
\hline Constipation & $0(0.0)$ & $1(0.9)$ & $0(0.0)$ & $>0.05$ & $>0.05$ & $>0.05$ \\
\hline Diarrhea & $14(12.6)^{\mathrm{a}}$ & $4(3.6)^{b}$ & $13(12.1)^{\mathrm{a}}$ & 0.014 & 0.019 & $>0.05$ \\
\hline Dysphagia & $1(0.9)^{\mathrm{a}}$ & $21(18.9)^{\mathrm{b}}$ & $0(0.0)^{\mathrm{a}}$ & $<0.001$ & $>0.001$ & $>0.05$ \\
\hline Chest pain & $1(0.9)^{\mathrm{a}}$ & $9(8.1)^{\mathrm{b}}$ & $0(0.0)^{\mathrm{a}}$ & 0.010 & 0.003 & $>0.05$ \\
\hline Darkened stool & $47(42.3)^{\mathrm{a}}$ & $51(45.9)^{\mathrm{a}}$ & $0(0.0)^{b}$ & $>0.05$ & $<0.001$ & $<0.001$ \\
\hline Black tongue & $12(10.8)^{\mathrm{a}}$ & $27(24.3)^{b}$ & $0(0.0)^{c}$ & 0.08 & $>0.001$ & $<0.001$ \\
\hline Vaginal discharge & $5(4.5)$ & $1(0.9)$ & $5(4.7)$ & $>0.05$ & $>0.05$ & $>0.05$ \\
\hline Headache & $7(6.3)$ & $1(0.9)$ & $4(3.7)$ & $>0.05$ & $>0.05$ & $>0.05$ \\
\hline Abdominal pain & $13(11.7)$ & $11(9.9)$ & $9(8.4)$ & $>0.05$ & $>0.05$ & $>0.05$ \\
\hline
\end{tabular}

Data are presented as $n(\%)$ unless otherwise indicated. See Table 1 footnote for abbreviations. ${ }^{*}$ Three $p$ values were used for 3 comparisons: RBAL vs. RBMT, RBMT vs. RAL, and RBAL vs. RAL, respectively. ${ }^{\text {a, b, c }}$ Protocols within a row without a common superscript differ.

lori (including urease, catalase, and lipase/phospholipase), preventing adhesion of $H$. pylori to surface epithelial cells, and inhibiting ATP synthesis and some membrane functions in $H$. pylori [20-22]. It has been reported that the addition of bismuth to standard triple therapy improved the eradication rate $[23,24]$. Equally important, Molina-Infante et al. [25] have reported that 2 nonbismuth quadruple therapies were effective in eradicating more than $90 \%$ of $H$. pylori infections in areas with a high clarithromycin resistance rate.

Levofloxacin, a broad-spectrum fluoroquinolone, was used in triple, quadruple, and sequential treatments [12$17,26,27]$ in which higher eradication rates of levofloxacin- than clarithromycin-containing therapy were found when used in a triple first-line regimen (7-10 days) [14, $15,17]$. In the present study, levofloxacin was used in triple and bismuth-containing quadruple therapies for 2 weeks, and triple therapy did not eradicate $H$. pylori. However, it has previously been shown that five days of levofloxacin-containing quadruple concomitant therapy was as effective and safe in eradicating $H$. pylori infection as 10 days of levofloxacin-containing sequential therapy [28]. Also, it has been reported that levofloxacin-contain- ing sequential therapy is more effective than clarithromycin-containing sequential therapy [29].

There are controversial findings on the efficacy of levofloxacin-containing treatments (with or without bismuth) compared with standard bismuth quadruple treatment as first- or second-line treatments. In a study from Hong Kong, levofloxacin-containing bismuth quadruple therapy was inferior to standard bismuth quadruple therapy in the treatment of resistant $H$. pylori infection (ITT eradication rates of 73 and $88 \%$ and PP eradication rates of 78 and $94 \%$, respectively, $p=0.030$ ). For those with a previous history of levofloxacin triple therapy, the eradication rates were 71 and $93 \%$, respectively $(p=0.139)$ [16].

The high eradication rates of RBAL (91.2\%) and RBMT $(92.5 \%)$ are consistent with those of Su et al. [17], who reported an eradication rate of $89.66 \%$ with levofloxacincontaining bismuth quadruple therapy, which was better than clarithromycin-containing bismuth quadruple therapy (80.25\% eradication rate) and levofloxacin-containing triple therapy ( $81.93 \%$ eradication rate).

In this study, the eradication rate of levofloxacin-containing triple therapy of less than $80 \%$ seemed to indicate that this was not a good option for first-line treatment. 
However, the addition of bismuth to the triple therapy improved the success rate to over $90 \%$. Since it is not possible to overcome levofloxacin resistance by increasing the dose and duration of the drug, standard quadruple therapy would seem to be the best choice as first-line treatment as an empiric protocol, with an eradication rate over $90 \%$.

In this study, considering the stable increase in levofloxacin and metronidazole resistance and negligible tetracycline resistance, quadruple therapies with bismuth seemed to be the best first-line treatment for $H$. pylori eradication in Turkey. However, the standard bismuth quadruple therapy caused side effects, but withdrawal rates were similar between the 3 groups. Also, the present study suggests that 14-day levofloxacin plus bismuth quadruple therapy can be used empirically in places where the levofloxacin resistance is known to be low. Further larger, multicenter studies with susceptibility test are needed to compare the eradication rates, and also compliance rates, of levofloxacin-containing bismuth quadruple therapy and levofloxacin-containing concomitant and sequential therapies with other treatment regimens.

The main limitation of our study is that no culture for $H$. pylori was performed and, therefore, antibiotic susceptibility/resistance rates were unknown.

\section{Conclusion}

All bismuth-containing quadruple therapies had higher eradication rates than levofloxacin-containing triple therapy. Hence, standard bismuth quadruple therapy should be considered the preferred first-line therapy if the antibiotic susceptibility is not known.

\section{References}

1 Cover TL, Blaser MJ: Helicobacter pylori in health and disease. Gastroenterology 2009; 136:1863-1873.

2 Kamboj AK, Cotter TG, Oxentenko AS: Helicobacter pylori: the past, present, and future in management. Mayo Clin Proc 2017;92:599604.

3 Suerbaum S, Michetti P: Helicobacter pylori infection. N Engl J Med 2002;347:1175-1186.

4 Malfertheiner P, Bazzolli F: Helicobacter $p y$ lori eradication with a capsule containing bismuth subcitrate potassium, metronidazole, and tetracycline given with omeprazole versus clarithromycin-based triple therapy: a randomized, open-label, non-inferiority, phase 3 trial. Lancet 2011;377:905-913.

5 Fischbach L, Evans EL: Meta-analysis: the effect of antibiotic resistance status on the efficacy of triple and quadruple first-line therapies for Helicobacter pylori. Aliment Pharmacol Ther 2007;26:343e57.

6 Malfertheiner P, Megraud F, O’Morain C, et al: Current European concepts in the management of Helicobacter pylori infection: the Maastricht Consensus Report. The European Helicobacter Pylori Study Group (EHPSG). Eur J Gastroenterol Hepatol 1997;9:1-2.

7 Zhao F, Wang J, Yang Y, et al: Effect of CYP2C19 genetic polymorphisms on the efficacy of proton pump inhibitor-based triple therapy for Helicobacter pylori eradication: a meta-analysis. Helicobacter 2008;13:532541.

8 Megraud F, Coenen S: Helicobacter pylori resistance to antibiotics in Europe and its relationship to antibiotic consumption. Gut 2013; 62:34-42.
9 Malfertheiner P, Megraud F: Management of Helicobacter pylori infection - the Maastricht V/Florence Consensus Report. Gut 2017;66: 6-30.

10 Lee ST, Lee DH, Lim JH, et al: Efficacy of 7-day and 14-day bismuth-containing quadruple therapy and 7-day and 14-day moxifloxacin-based triple therapy as second-line eradication for Helicobacter pylori infection. Gut Liver 2014;9:478-485.

11 Kocazeybek B, Tokman HB: Prevalence of primary antimicrobial resistance of $H$. pylori in Turkey: a systematic review. Helicobacter 2016;21:251-260.

12 Gisbert JP, Morena F: Systematic review and meta-analysis: levofloxacin-based rescue regimens after Helicobacter pylori treatment failure. Aliment Pharmacol Ther 2006;23:35-44.

13 Marin AC, McNicholl AG, Gisbert JP: A review of rescue regimens after clarithromycincontaining triple therapy failure (for Helicobacter pylori eradication). Expert Opin Pharmacother 2013;14:843-861.

14 Nista EC, Candelli M, Zocco MA, et al: Levofloxacin-based triple therapy in first-line treatment for Helicobacter pylori eradication. Am J Gastroenterol 2006;101:1985-1990.

15 Gisbert JP, Fernández-Bermejo M, MolinaInfante J, et al: First-line triple therapy with levofloxacin for Helicobacter pylori eradication. Aliment Pharmacol Ther 2007;26:495500.

16 Yee YK, Cheung TK, Chu KM, et al: Clinical trial: levofloxacin-based quadruple therapy was inferior to traditional quadruple therapy in the treatment of resistant Helicobacter $p y$ lori infection. Aliment Pharmacol Ther 2007; 26:1063-1067.
$17 \mathrm{Su}$ J, Zhou X, Chen H, et al: Efficacy of 1st-line bismuth-containing quadruple therapies with levofloxacin or clarithromycin for the eradication of Helicobacter pylori infection: a 1-week, open-label, randomized trial. Medicine (Baltimore) 2017;96:e5859.

18 Onder G, Aydin A, Akarca U, et al: High $\mathrm{He}$ licobacter pylori resistance rate to clarithromycin in Turkey. J Clin Gastroenterol 2007; 41:747-750.

19 Chey WD, Leontiadis GI, Howden CW, et al: ACG Clinical Guideline: treatment of Helicobacter pylori infection. Am J Gastroenterol 2017;112:212-239.

20 Keogan DM, Griffith DM: Current and potential applications of bismuth-based drugs. Molecules 2014;19:15258-15297.

21 Stratton CW, Warner RR, Coudron PE, et al: Bismuth mediated disruption of the glycocalyx-cell wall of Helicobacter pylori: ultrastructural evidence for a mechanism of action for bismuth salts. J Antimicrob Chemother 1999; 43:659-666.

22 Stoltenberg M, Martiny M, Sørensen K, et al: Histochemical tracing of bismuth in Helicobacter pylori after in vitro exposure to bismuth citrate. Scand J Gastroenterol 2001;36: 144-148.

23 Kekili M, Önal IK, Öcal S, et al: Inefficacy of triple therapy and comparison or two different bismuth-containing quadruple regimens as a first-line treatment option for Helicobacter pylori. Saudi J Gastroenterol 2016;22: 366-369. 
24 Sun Q, Liang X, Zheng Q, et al: High efficacy of 14-day triple therapy-based, bismuth-containing quadruple therapy for initial Helicobacter pylori eradication. Helicobacter 2010; 15:233-238.

25 Molina-Infante J, Romano M, FernandezBermejo $\mathrm{M}$, et al: Optimized nonbismuth quadruple therapies cure most patients with Helicobacter pylori infection in populations with high rates of antibiotic resistance. Gastroenterology 2013;145:121-128.
26 Liao J, Zheng Q, Liang X, et al: Effect of fluoroquinolone resistance on 14-day levofloxacin triple and triple plus bismuth quadruple therapy. Helicobacter 2013;18:373-377.

27 McNicholl AG, Gisbert JP: In H. pylori-infected patients second-line treatment with sequential levofloxacin therapy was more effective than levofloxacin triple therapy but was still suboptimal. Evid Based Med 2016;21: 144.
28 Federico A, Nardone G, Gravina AG, et al: Efficacy of 5-day levofloxacin-containing concomitant therapy in eradication of Helicobacter pylori infection. Gastroenterology 2012;143:55-61.

29 Romano M, Cuomo A, Gravina AG, et al: Empirical levofloxacin-containing versus clarithromycin-containing sequential therapy for Helicobacter pylori eradication: a randomised trial. Gut 2010;59:1465-1470. 